\title{
Análisis prospectivo de la calidad del servicio al cliente en la industria hotelera de la ciudad de Panamá del 2018 al 2023
}

\author{
Miroslava Alzamora de Zárate ${ }^{1 *}$ \\ 1Universidad Católica Santa María la Antigua, Facultad de Negocios, Panamá, República de Panamá
}

*Autor para correspondencia. Email: malzamora@usma.ac.pa

Recibido: 23 de octubre de 2019

Aceptado: 20 de noviembre de 2019

\begin{abstract}
Resumen
Los diagnósticos analizados y detallados en este trabajo, indican que tanto el sector turismo como la industria hotelera están pasando por momentos bastantes difíciles los cuales han venido disminuyendo el nivel de su negocio y su competitividad en el mercado, ocasionando el despido de personal y el cierre de operaciones de muchas empresas en Panamá. Se realizaron entrevistas a varios actores relevantes del sector turismo en Panamá donde la pregunta principal fue identificar los ocho (8) problemas más sobresalientes con relación al servicio al cliente y cómo visualizar la solución de los mismos. Se analizó de qué manera, desde el punto de vista de cada uno de ellos, se podrían mejorar estas debilidades y problemáticas. Los resultados obtenidos evidenciaron que la calidad del servicio al cliente en las empresas hoteleras presenta carencias y debilidades como: Baja capacidad de gestión y supervisión por parte de los mandos medios. Baja satisfacción laboral y motivación por parte de los colaboradores. Escaso personal capacitado con dominio del idioma inglés. Falta de interés en trabajar en el sector turismo en horarios rotativos y laborar en días festivos. Los propietarios no ofrecen oportunidades de capacitación para desarrollo de habilidades y destrezas del personal. Falta de interés en ofrecer un servicio y atención al cliente en forma eficiente. Deficiente cultura turística. Falta de incentivos y de promoción laboral. Entre los métodos utilizados está el Análisis Estructural que consiste en interrelacionar las variables en un cuadro de doble entrada Matriz de Análisis Estructural. Esta matriz se elabora de manera cualitativa (existencia o no de relaciones), pero también cuantificable. De esta forma, se distinguen varias intensidades de relaciones directas: fuertes, medias, débiles y potenciales. Luego se procede a la búsqueda de las variables claves utilizando Sistemas y Matrices de Impactos Cruzados (SMIC) y Multiplicación Aplicada a una Clasificación (MICMAC). Cada variable lleva aparejado un indicador de motricidad y dependencia.
\end{abstract}

Palabras clave: Análisis prospectivo, servicio, calidad, hotelería, turismo.

\section{Abstract}

The diagnoses analyzed and detailed in this work, indicate that both the tourism sector and the hotel industry are going through quite difficult times when they have been decreasing the level of their business and their competitiveness in the market, causing the dismissal of staff and cessation of business operations for many companies in Panama. 
Interviews were conducted to several relevant actors in the tourism sector in Panama where the main question was to identify the eight most outstanding problems in relation to customer service and how to visualize their solution. It was analyzed how, from the point of view of each of them, these weaknesses and problems could be improved. The results obtained showed that the quality of customer service in hotel companies has shortcomings and weaknesses such as: Low management capacity and supervision by middle managers. Low job satisfaction and motivation by employees. Poor trained personnel with mastery of the English language. Lack of interest in working in the tourism sector for rotating schedules and working on holidays. The owners do not offer training opportunities for the development of skills and abilities of the staff. Lack of interest in offering a customer service in an efficient way. Poor tourist culture. Lack of incentives and job promotion. Among the methods used is the Structural Analysis Method will be used, which consists of interrelating the variables in a double entry box Structural Analysis Matrix. This matrix is elaborated in a qualitative way (existence or not of relations), but also quantifiable. In this way, several intensities of direct relationships are distinguished: strong, medium, weak and potential. Then we proceed to search for the key variables using Cross Impact Matrix (CIM) and Multiplication Applied to a Classification (MICMAC). Each variable has an indicator of motor skills and dependence.

Keywords: Prospective analysis, service, quality, hotel industry, tourism.

\section{Introducción}

Panamá ha situado el turismo como uno de los sectores económicos prioritarios del país. Durante el 2017 tuvo un crecimiento del 4\% con relación al 2016 lo que representó aproximadamente 2.2 millones de visitantes.

Flujos de esta magnitud colocan al turismo como uno de los principales motores de la economía panameña, con un notable potencial de crecimiento, a partir de que el país aparece muy bien posicionado en los rankings globales de turismo, aun dentro del marco de indicadores de desaceleración económica. Entre las mediciones positivas más recientes, el Índice de Competitividad de Viajes y Turismo, auspiciado por el Foro Económico Mundial, señala que en 2017 Panamá se colocó en el tercer lugar entre los destinos favoritos para el turismo de América Latina, superado solo por gigantes como México y Brasil (WEF, 2017). De igual manera, la revista Forbes en español incluyó a Panamá, mediante su Global Destination Cities Index, entre los diez (10) sitios turísticos latinoamericanos más populares para 2018. En esta clasificación se toman en cuenta factores como la variedad de los paisajes, el acceso a recursos naturales y culturales, entre otros atractivos (Mi Diario, 2018).

El servicio excelente al cliente debe estar presente en todos los aspectos del negocio en donde haya alguna interacción con el ser humano. Debe ir desde el saludo del personal encargado de la seguridad del negocio hasta los altos niveles jerárquicos de la organización.

Los clientes son cada vez más exigentes, ya no solo buscan calidad y precio, sino también una buena atención, un ambiente agradable, una rápida atención, un trato personalizado y lo más importante, vivir una experiencia inolvidable que quieran repetir. 
Por otro lado, y según el Boletín Estadístico 2017, preparado por el Departamento de Estadística de la Autoridad de Turismo de Panamá (ATP, 2017), el turismo juega un papel muy importante en la determinación de las tendencias del ciclo económico general y de varias actividades económicas específicas y como tal la actividad turística para el año 2017 encabeza el primer lugar dentro de los principales generadores de divisas en el Sector de la Economía.

El turismo aportó 6,856.3 millones de balboas que corresponde a la suma del renglón viaje de la Balanza de pagos y al Transporte Internacional, la Zona Libre de Colón con 1,218.2 millones del Valor Neto Exportado y el Canal de Panamá (peaje) con 2,316.3 millones de balboas de los Peajes Cobrados. El gasto promedio por turista para el 2016 fue de 2,723 balboas y un gasto diario de 340 balboas (incluye pasaje internacional).

La evolución del turismo en Panamá ha crecido significativamente en los últimos diez (10) años reflejando una tasa media de crecimiento del 8\% y una participación del Turismo en el Producto Interno Bruto de 11\% sobre el año 2016.

En cuanto a la industria hotelera aporta enormes beneficios a la economía de distintos países y muchas veces situándose como uno de los pilares más importantes de estos países. Es por esta razón que, la gestión que se realiza para ofertar calidad en el servicio en los hoteles se convierte en uno de los elementos con mayor relevancia en el desarrollo del turismo, es decir que, al generarse calidad, sinónimo de satisfacción en los viajeros, se produce sostenibilidad de la ciudad, puesto que se da un incremento en el número de personas que visitan el destino turístico.

Panamá ha crecido enormemente como destino en los últimos cuatro (4) años. Las multimillonarias inversiones que están realizando estas cadenas de hoteles internacionales muestran no sólo el nivel de confianza en el desarrollo turístico del país sino también están creando las condiciones para satisfacer las exigencias de un número de visitantes cada vez mayor. De acuerdo al Registro Nacional de Turismo de la ATP (ATP, 2018b), muestra un total de 2,358 empresas turísticas registradas, de las cuales 912 corresponden a hospedaje.

Panamá cuenta con muchas y grandes cadenas internacionales de hoteles como Hilton, Starwood, Sheraton y Trump, Marriott, Holiday Inn entre otros, quienes han inaugurado modernas y sofisticadas propiedades en miras de cubrir la creciente demanda en el país.

Según Resumen Estadístico Enero a Junio 2018 de la ATP (ATP, 2018a), indica que el número de habitaciones existente hasta el año 2017, fue de 32,530. El Porcentaje de Ocupación Promedio en el año 2017, fue de $47.09 \%$. De enero a mayo de 2018, este porcentaje de ocupación hotelera fue de 46.5\%, el cual representa una disminución de 5.4\% en comparación con el mismo periodo de 2017.

La industria hotelera ha evolucionado gracias al mayor atractivo con el que cuenta Panamá: El Canal. Esto ha causado que muchas personas nos visiten y también ha traído consigo un crecimiento en la ocupación hotelera. 


\section{Materiales y métodos}

La presente investigación es de tipo descriptiva y de diseño no experimental del tipo transversal. La investigación busca evaluar las opiniones de los diferentes expertos del sector turismo sobre la calidad del servicio al cliente en Panamá.

Dentro de la investigación no experimental, se seleccionó la aproximación transeccional descriptiva, ya que se recolecta la información, para describir el estado de las variables, en su ambiente natural, en un solo momento y en un momento dado.

Tomando de referencia, el Análisis Diagnóstico General del Turismo en Panamá-Plan Maestro de Turismo Sostenible de Panamá 2007-2020, los principales actores del sector turismo, por la iniciativa privada, serían: Asociación de Líneas Aéreas de Panamá (ALAP), Asociación Panameña de Hoteles (APATEL), Asociación de Pequeños Hoteles de Panamá (HOPPAN), Asociación Panameña de Operadores de Turismo (APOTUR), Asociación Panameña de Agencias de Viajes y Turismo (APAVIT), Asociación Nacional de Arrendadores de Vehículos (ANAV), Asociación de Restaurantes y Afines de Panamá $\square$ (ARAP) y Buró de Convenciones y Visitantes de Panamá $\square$ (BCVP).

Integraron también la muestra, algunos entes públicos y organismos ramales involucrados en el sector turismo y que aportaron al presente estudio, entre ellos: Cámara de Turismo de Panamá (CAMTUR), Asociación Panameña de Hoteles (APATEL), Autoridad de Turismo de Panamá (ATP), conocido antes como Instituto Panameño de Turismo (IPAT), Asociación Panameña de Agencias de Viajes y Turismo (APAVIT). El total de los gremios entrevistados representan el 50\% de los gremios indicados el Análisis Diagnóstico General del Turismo en Panamá-Plan Maestro de Turismo Sostenible de Panamá 2007-2020.

Adicionalmente, se tomaron en consideración algunas instituciones académicas universitarias que brindan la carrera de turismo como lo son la Universidad Católica Santa María la Antigua (USMA), Universidad Interamericana de Panamá (UIP), Universidad del Istmo (UDI), Universidad Latina de Panamá, ISAE, Universidad Especializada de las Américas (UDELAS) y Universidad de Panamá (UP). Entrevistamos a seis (6) expertos académicos de algunas de estas instituciones como lo son la USMA, UIP, ISAE y la UP, lo que representa un 57\% del total de instituciones de educación superior con ofertas formativas para el sector turístico.

La selección de la muestra se realizó tomando en consideración que es importante que desde que se eligen a los expertos, éstos realmente cuenten con la experticia en la temática del estudio, siendo MICMAC (Godet, 1991, 1999) un método mixto cuantitativo y cualitativo, lo que le permite al decisor establecer cuáles son aquellas variables que por su influencia afectan a todo el sistema y con relación a esto, finalmente tomar las acciones que resulten más adecuadas.

La recopilación de la información se llevó a cabo a partir de fuentes primarias. Se emplearon entrevistas directas (Hernández Sampieri et al., 2014), de tipo semi-estructurado, usando una guía de preguntas a doce (12) actores del sector turismo identificados, con preguntas de tipo cerradas y abiertas, durante los meses de enero y febrero de 2018, para conocer al menos ocho (8) problemas que 
consideran se da en el tema del servicio al cliente en la industria hotelera de Panamá. Estas variables fueron también definidas con la ayuda de los expertos entrevistados.

Posteriormente, con la ayuda de estos expertos se clasificaron las variables mediante la matriz de la relación entre las variables y se realizó el análisis estructural (Godet, Op.cit), el cual consistió en el cruce de cada una de ellas en una matriz de impacto que definió la dependencia y motricidad de las mismas y permitió determinar las variables claves. Seguidamente, se procedió a elaborar la matriz de análisis estructural, en la que se sitúan las variables en cuatro (4) zonas: la zona de poder, la zona de conflicto, la zona de salida y la zona de indiferencia.

Los expertos entrevistados definieron, de acuerdo a su criterio, los actores que juegan un papel predominante y que se consideran tienen mayor participación en sector turístico de Panamá.

Posteriormente se procedió a clasificar variables y actores claves para determinar su nivel de influencia y dependencia, buscando valorar las relaciones de fuerza entre los actores y estudiar sus influencias y dependencias.

Para realizar el análisis del juego de actores (Godet, Íbid.), se utilizó una matriz en la que se ubicaron las variables claves ya definidas y se confeccionó una lista de los mismos y sus objetivos pertinentes, buscando definir la posición de cada uno, así como las posibilidades de alianzas y conflictos entre ellos, las estrategias y los eventos a considerar.

Con los resultados del análisis estructural y del juego de actores, se procedió al planteamiento de hipótesis, tomando como referencia la probabilidad de ocurrencia de eventos asociados a la variable y objetivos estratégicos. Posteriormente, se consultó a los expertos en torno a la probabilidad de ocurrencia de las hipótesis. Las respuestas obtenidas fueron la base sobre la cual se elaboraron los escenarios del futuro considerando que puedan ser optimistas, moderados o pesimistas.

\section{Resultados}

\subsection{Entrevistas.}

La entrevista realizada a representantes de estas instituciones busca, en primera instancia, identificar las principales debilidades y deficiencias que enfrenta el sector hotelero de la ciudad de Panamá, en lo relacionado a la atención de los clientes, cómo visualizan ellos la solución de estos problemas y quiénes deberían intervenir para la solución de estos problemas.

La Asociación Panameña de Hoteles (APATEL), según su Directora Ejecutiva, Lic. Ana María Sanchíz, mencionó ocho (8) problemas que enfrenta este sector: Baja capacidad de gestión y supervisión. Baja satisfacción laboral y motivación necesarios para atención al visitante en busca de experiencias memorables. Escaso personal capacitado con dominio del idioma inglés. Falta de formación en servicio al cliente. Falta de interés en trabajar en turismo ya que requiere que los colaboradores laboren en turnos rotativos, domingos y en días festivos. Poco interés en ofrecer un servicio y atención al cliente. Cultura turística deficiente, con poco conocimiento sobre el entorno. Propietarios no ofrecen 
oportunidades a empleados de capacitaciones en atención y servicio al cliente excelentes para desarrollar y mejorar habilidades y aptitudes.

La Lic. Sara Sánchez, exfuncionaria de la Autoridad de Turismo de Panamá (ATP), mencionó ocho (8) problemas que ha identificado con relación a esta problemática: Actitud personal, baja autoestima de los prestadores de servicio que los hace reaccionar negativamente. Desconocimiento de los elementos básicos del servicio al cliente. Falta de motivación por parte de las empresas empleadoras. Falta de supervisión, entendiendo que la persona que supervisa debe tener conocimiento de los temas. No dominio del idioma del visitante, este aspecto puede superarse con una buena actitud. Poca disposición a la sonrisa. Poca atención en la escogencia del personal que hará frente al visitante y falta de capacitación. No hay cultura de servicio y el entorno social en el que se desarrolla la empresa tiene mucha injerencia en la disposición o no, de brindar un buen servicio.

En el tema del servicio al cliente, expertos de la Asociación Panameña de Agencias de Viajes y Turismo (APAVIT), señalaron que la materia de servicio al cliente, impartida en las universidades, debe ser reforzada con temas adicionales y debe ser incluida en todas las carreras. En estos momentos, es impartida para los estudiantes de hotelería, solamente, señalándose que es importante que en todas las carreras se le dé el debido énfasis a esta materia.

Los expertos del ámbito académico nos ayudaron a definir los ocho (8) problemas más sobresalientes en el tema del servicio al cliente en la industria hotelera de Panamá: Baja capacidad de gestión y supervisión por parte de los mandos medios. Baja satisfacción laboral y motivación por parte de los colaboradores. Escaso personal capacitado con dominio del idioma inglés. Propietarios no ofrecen oportunidades de capacitación para desarrollo de habilidades y destrezas del personal. Falta de interés en trabajar en turismo por horarios rotativos y labores en días festivos. Poco interés en ofrecer un servicio y atención al cliente. Deficiente cultura turística. Falta de incentivos y de promoción para que el colaborador se sienta motivado.

A la vez, mencionaron otros problemas a los que nos enfrentamos: Falta de formación básica en este tema desde los bachilleratos. Bajos salarios. Resulta muy difícil ascender jerárquicamente. Poco incentivo por parte del gobierno en el sector turismo. Falta de involucramiento de toda la empresa en el servicio. Problemas de empoderamiento y que debe ser considerado y convertirse en un tema de política de Estado.

Los expertos Antonio Alfaro, Alberto Quirós Jaén y Jaime Dreyfuss, de la Cámara Panameña de Turismo (CAMTUR), señalaron que la clave de poder atender estas debilidades que enfrenta el sector turismo relacionado a la atención a los clientes se enfoca en brindar capacitaciones relacionadas a este tema y que la academia es un actor clave para ayudar a cumplir con este reto.

Estos expertos compartieron los resultados de las mesas de trabajo de CONATUR (Convención Nacional de Turismo), realizada en junio del año 2017. A continuación, se mencionan los resultados obtenidos en cada una de las mesas establecidas para lograr algunas estrategias que favorezcan al 
sector: Identificar la necesidad de reforzar el manejo de otros idiomas fue un punto que se conversó a lo largo del desarrollo del tema en cada pregunta y mesa. Establecer programas para reforzar los valores positivos en la sociedad y a todos los niveles. Establecer centros operativos de capacitación con su equipo de facilitadores, docentes de universidades, instructores contratados por ATP e INADEH. Considerar que el sector privado debe invertir en la capacitación de sus colaboradores a la par de contar con acciones a nivel nacional. Crear conciencia sobre la autoformación. Lema que surgió en una mesa: "Cambiar de Turismo a Hospitalidad". Medir los resultados por pare de la entidad / persona que capacita. Implementar plan de capacitación tanto por parte de la empresa pública como la privada.

Señalaron también: Establecer planes de incentivos dentro de reglamento interno de las empresas. Fortalecer los servicios turísticos en Idiomas. Capacitar a los guías de turismo en temas de Historia y considerarlos como anfitriones. Reforzar los temas de folklore, cultura y tradiciones. Capacitar en temas de inteligencia emocional a los colaboradores, y reforzar los temas de valores en campañas visuales y de radio. Proponer que las empresas deben tener normas escritas y motivaciones a sus colaboradores. Reforzar tema de capacitaciones en sector público en temas relacionados con la atención al cliente. (Policía, migración, aduana, entre otros).

Promover el trabajo en equipo entre el sector público y el sector privado. Incorporar en las escuelas los programas de cultura turística. Motivar la reingeniería en el sistema educativo. Promover y promocionar programas existentes en temas de formación. Crear plataforma de información, con datos actualizados. Hacer cumplir las normas y establecer programas continuos de capacitación de parte de los propietarios de bares y restaurantes.

\subsection{Análisis Estructural y de Escenarios.}

Para este procedimiento se definieron las variables del sistema, mediante la identificación de los ocho (8) problemas más sobresalientes identificados en el proceso de entrevistas con los actores del sector turismo identificados. Como se puede observar en la tabla $\mathrm{N}^{0} 1$, el análisis se centra en los ocho (8) problemas que fueron identificados durante las entrevistas.

Una vez identificados los problemas o variables, se procede a dar una definición de cada uno de ellos, con la ayuda de los expertos entrevistados, para poderlos entender de una mejor manera. 
Tabla $\mathbf{N}^{\circ} 1$

MATRIZ DE RELACIONES ENTRE VARIABLES

\begin{tabular}{|c|l|c|c|c|c|c|c|c|c|c|}
\hline \multicolumn{1}{|c|}{ NOMBRE } & V1 & V2 & V3 & V4 & V5 & V6 & V7 & V8 & $\begin{array}{c}\text { TOTAL } \\
\text { MOTRICIDAD }\end{array}$ \\
\hline V1 & $\begin{array}{l}\text { Baja capacidad de gestión y supervisión por parte de los } \\
\text { mando medios }\end{array}$ & $\mathrm{X}$ & 3 & 1 & 3 & 0 & 3 & 0 & 3 & 13 \\
\hline V2 & $\begin{array}{l}\text { Baja satisfacción laboral y motivación por parte de los } \\
\text { colaboradores }\end{array}$ & 3 & $\mathrm{X}$ & 0 & 3 & 0 & 3 & 0 & 3 & 12 \\
\hline V3 & Escaso personal capacitado con dominio del idioma Inglés & 0 & 0 & $\mathrm{X}$ & 0 & 0 & 2 & 0 & 0 & 2 \\
\hline V4 & $\begin{array}{l}\text { Propietarios no ofrecen oportunidades de capacitación } \\
\text { para el desarrollo de habilidades y destrezas del personal }\end{array}$ & 3 & 3 & 3 & $X$ & 2 & 3 & 0 & 3 & 17 \\
\hline V5 & $\begin{array}{l}\text { Falta de interés en trabajar en turismo por horarios } \\
\text { rotativos y labores en días festivos }\end{array}$ & 0 & 2 & 3 & 3 & $X$ & 3 & 3 & 3 & 17 \\
\hline V6 & Poco interés en ofrecer un servicio y atención al cliente & 3 & 3 & 3 & 3 & 3 & $X$ & 2 & 3 & 20 \\
\hline V7 & Deficiente cultura turistica & 0 & 2 & 3 & 3 & 1 & 3 & $X$ & 0 & 12 \\
\hline V8 & $\begin{array}{l}\text { Falta de incentivos y de promoción para que el } \\
\text { colaborador se sienta motivado }\end{array}$ & 3 & 3 & 0 & 3 & 1 & 3 & 0 & X & 13 \\
\hline $\begin{array}{c}\text { TOTAL } \\
\text { DEPENDENCIA }\end{array}$ & & 12 & 16 & 13 & 18 & 7 & 20 & 5 & 15 & 106 \\
\hline
\end{tabular}

$\begin{array}{ll}3 & \text { Influencia directa fuerte: } \\ 2 & \text { Influencia directa media: } \\ 1 & \text { Influencia directa débil o potencial: } \\ 0 & \text { Influencia nula: }\end{array}$

\begin{tabular}{|c|c|c|c|c|c|c|c|c|}
\hline RESUMEN & V1 & V2 & V3 & V4 & V5 & V6 & V7 & V8 \\
\hline MOTRICIDAD & 13 & 12 & 2 & 17 & 17 & 20 & 12 & 13 \\
\hline DEPENDENCIA & 12 & 16 & 13 & 18 & 7 & 20 & 5 & 15 \\
\hline
\end{tabular}

Los resultados obtenidos a partir de la opinión expresada por los diferentes expertos a los que se le realizó la entrevista, se muestran en la Tabla $\mathrm{N}^{\circ} 1$, denominada matriz de relaciones entre problemas, donde los valores X e Y, representan la intensidad de la relación entre cada variables o problemas. Adicionalmente se presentan la suma de los valores por fila para representar el grado de motricidad de una variable sobre las otras; y la sumatoria de los valores por columna para representar el grado de dependencia de cada variable.

Se consideró la siguiente escala:

3 Influencia directa fuerte

1 Influencia directa débil o potencial $0 \quad$ Influencia nula

Las tres (3) variables que mostraron mayor motricidad fueron V6: 20, V4: 17, V5: 17.

\begin{tabular}{|l|l|}
\hline V6 & Poco interés en ofrecer un servicio y atención al cliente \\
\hline V4 & $\begin{array}{l}\text { Propietarios no ofrecen oportunidades de capacitación para el desarrollo de } \\
\text { habilidades y destrezas del personal }\end{array}$ \\
\hline V5 & $\begin{array}{l}\text { Falta de interés en trabajar en turismo por horarios rotativos y labores en días } \\
\text { festivos }\end{array}$ \\
\hline
\end{tabular}


Las tres (3) variables que mostraron mayor dependencia fueron V6:20, V4: 18, V2:16.

\begin{tabular}{|l|l|}
\hline V6 & Poco interés en ofrecer un servicio y atención al cliente \\
\hline V4 & $\begin{array}{l}\text { Propietarios no ofrecen oportunidades de capacitación para el desarrollo de } \\
\text { habilidades y destrezas del personal }\end{array}$ \\
\hline V2 & Baja satisfacción laboral y motivación por parte de los colaboradores \\
\hline
\end{tabular}

La información obtenida en la tabla anterior permite calcular los índices de motricidad y dependencia para cada problema, así como ubicar estas relaciones en un diagrama de dispersión con el objeto de apreciar, gráficamente, la posición estructural de cada problema. Los cálculos resultantes y el diagrama de dispersión se muestran en el gráfico $\mathrm{N}^{\circ} 1$.

\section{Gráfico $\mathbf{N}^{\circ} 1$}

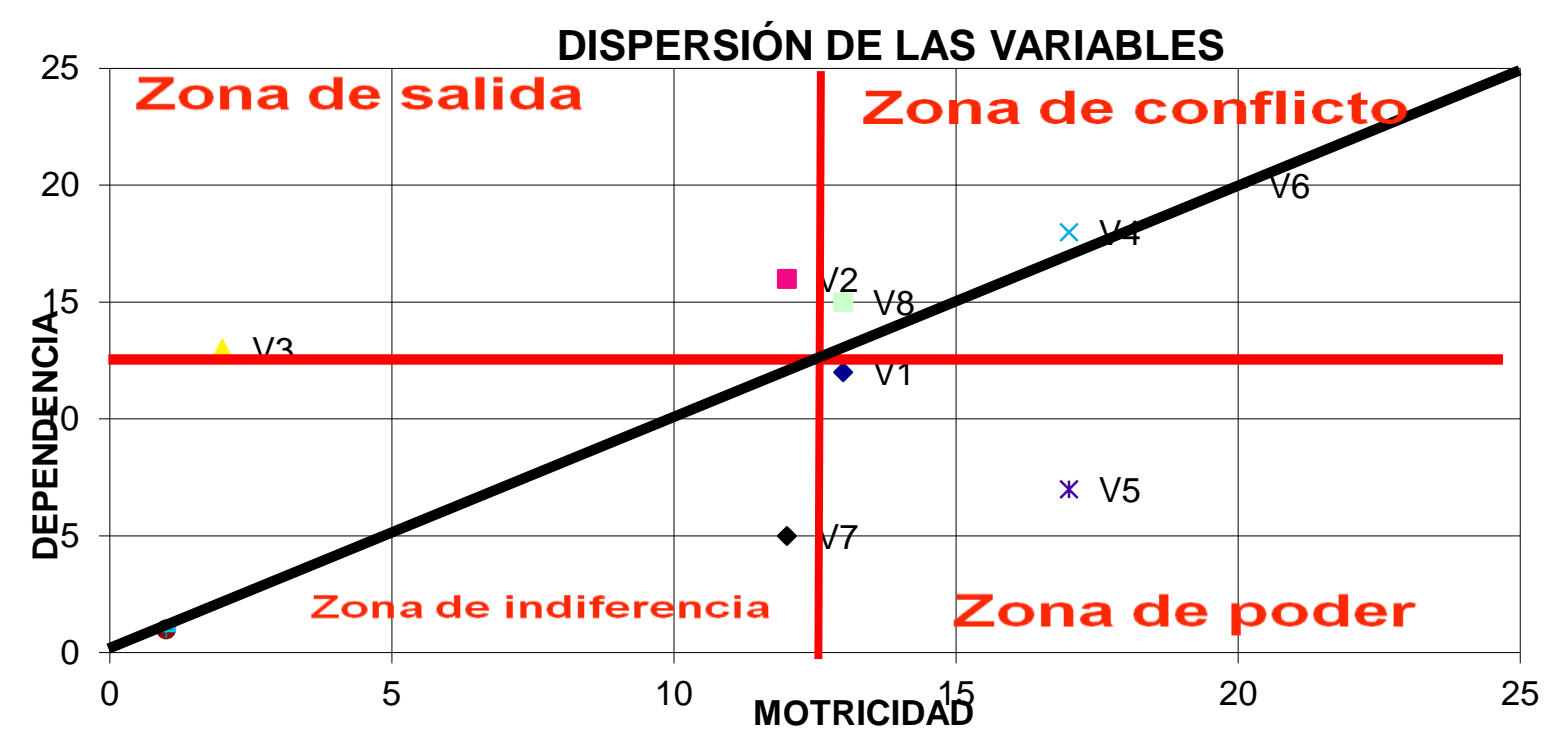

Los ocho (8) problemas que se presentan en el tema del servicio al cliente en la industria hotelera de Panamá, muestran diferentes posiciones relativas en el plano de la motricidad y la dependencia, como se observa en el gráfico siguiente. El objetivo del análisis es interpretar el conjunto de problemas en función de su posición en cada uno de los cuadrantes los cuales representan las zonas de poder, conflicto, salida e indiferencia.

Como se puede observar, en la zona de conflicto se encuentran las variables: V2, V4, V6 y V8, en la zona de poder se encuentran las variables: V1, V5, en la zona de salida se encuentra la variable V3, y en entre la zona de indiferencia y la zona de poder se encuentra la variable V7. Es de interés enfocarse en las variables que se encuentran en la zona de conflicto en vista de que es la que muestra una alta motricidad y alta dependencia. Estas variables son muy influyentes y son también altamente vulnerables, influyen sobre las restantes, pero a su vez son influidas por ellas. 
En esta tabla $\mathrm{N}^{\circ} 2$ se ha evaluado el nivel de influencia o de dependencia que tienen estos actores. De acuerdo a los resultados se puede concluir que, si hay conexión e identificación entre los actores, a fin de resolver o aportar a los problemas que identificamos. Esto es de valorar ya que, si hay vinculación e integración entre todos estos actores, que son vitales para el sector turismo y para la industria hotelera, se podrá llegar a acuerdos, recomendaciones y/o acciones que brinden resultados inmediatos a la mejora del servicio al cliente en ciudad de Panamá, en los próximos cinco (5) años venideros.

\section{Tabla $\mathbf{N}^{\circ} 2$}

\section{CALIFICACIÓN DE ACTORES}

\begin{tabular}{|c|c|c|c|c|c|c|c|c|c|}
\hline & ACTORES & A1 & A2 & A3 & A4 & A5 & A6 & A7 & $\begin{array}{c}\text { TOTAL } \\
\text { INFLUENCIA }\end{array}$ \\
\hline A1 & ATP & $\mathrm{X}$ & 1 & 0 & 1 & 1 & 1 & 1 & 5 \\
\hline A2 & UNIVERSIDADES PARTICULARES/PÚBLICAS & 1 & $\mathrm{X}$ & 1 & 2 & 2 & 1 & 1 & 8 \\
\hline A3 & INADEH & 2 & 2 & $\mathrm{X}$ & 3 & 3 & 3 & 3 & 16 \\
\hline A4 & CAMTUR & 1 & 2 & 0 & $\mathrm{X}$ & 1 & 1 & 1 & 6 \\
\hline A5 & CCIAP & 2 & 2 & 1 & 1 & $\mathrm{X}$ & 1 & 2 & 9 \\
\hline A6 & APATEL & 2 & 1 & 0 & 1 & 2 & $\mathrm{X}$ & 1 & 7 \\
\hline \begin{tabular}{|c|} 
A7 \\
\end{tabular} & EMPRESAS HOTELERAS & 1 & 2 & 0 & 1 & 1 & 1 & $\mathrm{X}$ & 6 \\
\hline $\begin{array}{c}\text { TOTAL } \\
\text { DEPENDENCIA }\end{array}$ & & 9 & 10 & 2 & 9 & 10 & 8 & 9 & 57 \\
\hline
\end{tabular}

El actor Ai puede cuestionar la existencia del actor $\mathrm{A}$

El actor Ai puede cuestionar la misión del actor $\mathrm{Aj}$

El actor Ai puede cuestionar los proyectos del actor $\mathrm{Aj}$

El actor Ai puede cuestionar de manera limitada (durante algún tiempo o en algún caso en concreto) la operatividad del actor $\mathrm{A} j$

El actor Ai no tiene ninguna influencia sobre el actor $\mathrm{Aj}$

\begin{tabular}{|c|c|c|c|c|c|c|c|c|}
\hline RESUMEN & A1 & A2 & A3 & A4 & A5 & A6 & A7 & TOTALES \\
\hline INFLUENCIA & 5 & 8 & 16 & 6 & 9 & 7 & 6 & 57 \\
\hline DEPENDENCIA & 9 & 10 & 2 & 9 & 10 & 8 & 9 & 57 \\
\hline
\end{tabular}

Cabe destacar, que el INADEH muestra un alto nivel de influencia sobre los demás actores, lo que le permite que se incorpore en los proyectos de los otros actores y sobre todo más gestiones de formación técnica que en funciones administrativas. Sin embargo, de momento los resultados indican que INADEH como actor tiene poca dependencia de los demás, lo que le permite generar mayores aportes.

\section{Gráfica $\mathrm{N}^{\circ} 2$}

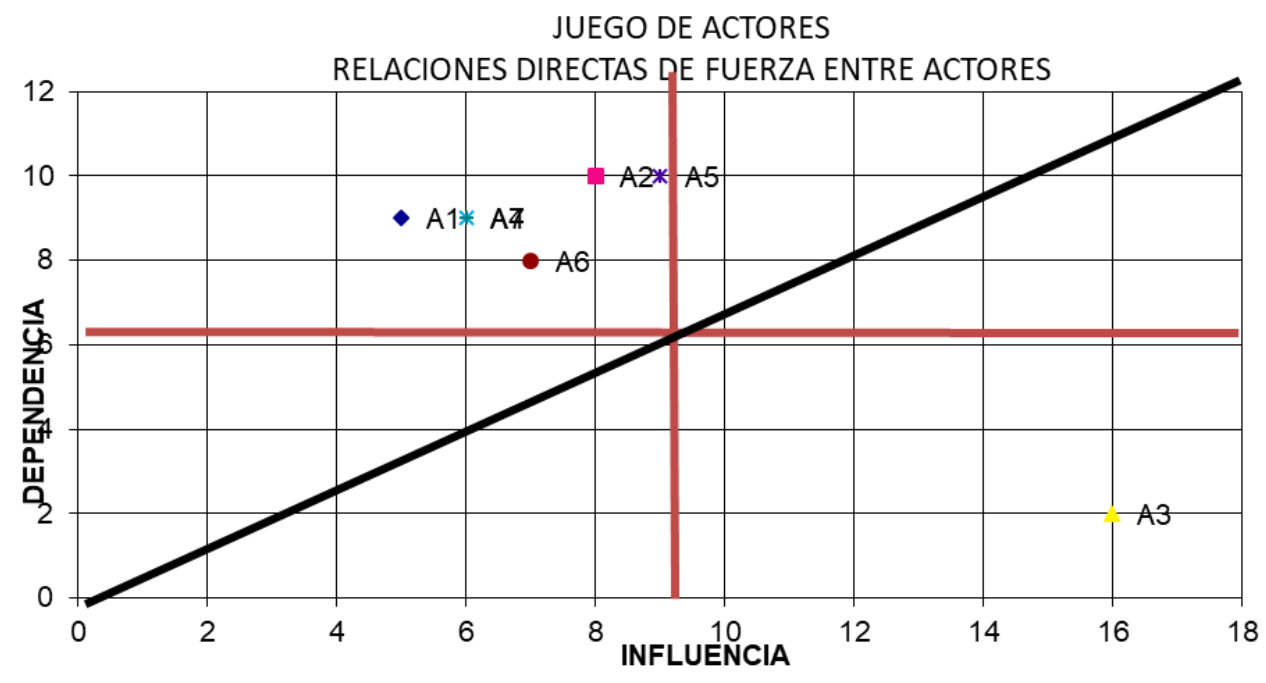


La gráfica $\mathrm{N}^{\circ} 2$ muestra las relaciones directas de fuerza entre los diferentes actores identificados. Como se puede observar, en la zona de mayor influencia está el actor A3 INADEH, sin embargo, se encuentra en el cuadrante de no interactuación con los demás actores al no contar con una relación de dependencia. Ello nos muestra que su actuación podrá contribuir debidamente orientada a mayor beneficio de desarrollo y crecimiento de los demás actores.

Otro aspecto a resaltar es el actor A5 CCIAP que relaciona una dependencia importante pero no interactuante con los demás actores: ATP, Universidades particulares y públicas, CAMTUR, APATEL y empresas hoteleras. A su vez, la gráfica nos identifica una necesidad de integración del actor A5 CCIAP para tener mayor sinergia y trabajo en equipo más directo con las fuerzas vivas del turismo.

Podemos notar en el cuadrante superior izquierdo que entre A1, A4, A7 y A6 destacan una dependencia vinculante para el desarrollo de la industria del turismo como hotelera en Panamá. También se refleja una importante relación participativa de las universidades particulares como públicas de apoyo al desarrollo del turismo en Panamá. Últimamente, con marcados programas académicos enfocados a ésta área, principalmente, a la dirección del negocio.

\section{Tabla $\mathbf{N}^{\circ} 3$}

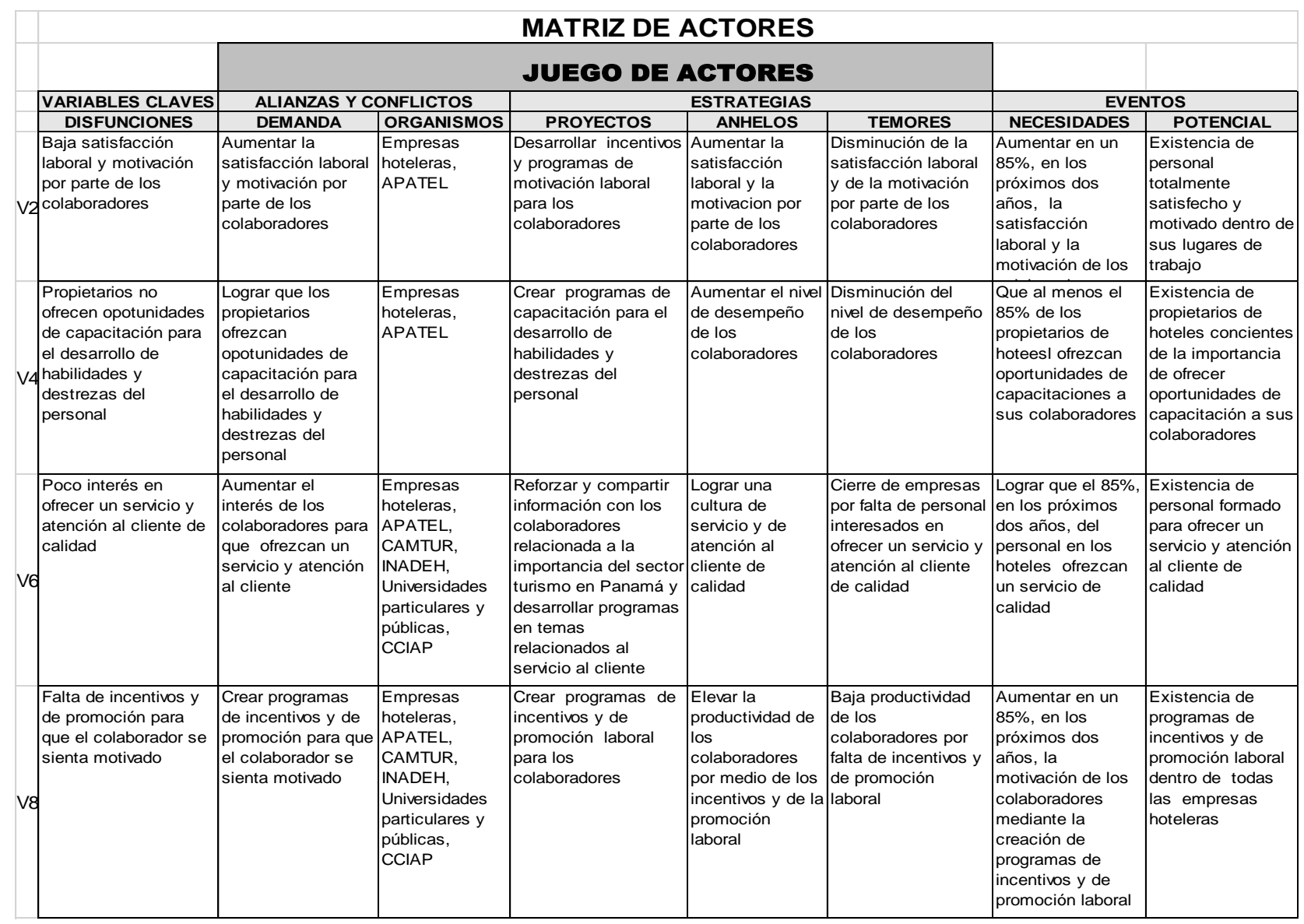


Como siguiente paso, se tomaron las variables claves que se obtienen mediante el análisis estructural y que se ubicaron en el área de conflicto que se muestra en la gráfica $\mathrm{N}^{\circ} 1$. Ellas son: V2=Baja satisfacción laboral y motivación por parte de los colaboradores. V4= Propietarios no ofrecen oportunidades de capacitación para el desarrollo de habilidades y destrezas del personal. V6= Poco interés en ofrecer un servicio y atención al cliente de calidad. V8= Falta de incentivos y de promoción para que el colaborador se sienta motivado y se establecen las alianzas y los conflictos que se presentan entre los actores, en vista de que es conocido que los problemas dan lugar a demandas de un actor a otro y esta situación, a su vez, puede generar posibles alianzas y conflictos entre ellos, tal cual se muestra en esta tabla.

A su vez, el establecimiento de estas alianzas y conflictos nos ayudan a conocer cuáles son los organismos e instituciones que intervienen en el juego de actores. Adicionalmente, en esta misma matriz se definen los proyectos, anhelos y temores correspondientes a cada actor, se construyen los eventos a partir de las variables claves de la forma más precisa y clara y seguidamente, las potencialidades se redactan a partir de los anhelos correspondientes.

\section{Tabla $\mathbf{N}^{\circ} 4$}

\section{EL MÉTODO DE ESCENARIO} FORMULACIÓN DE LAS HIPÓTESIS

\section{Problema Clave}

1

Baja satisfacción laboral y motivación por parte de los colaboradores

H1 = En los próximos 5 años el Sector Hotelero contará con una alta satisfacción laboral y motivación por parte de los colaboradores.

$2 \quad$ Propietarios no ofrecen oportunidades de capacitación para el desarrollo de habilidades y destrezas del personal.

$\mathrm{H} 2=$ Todos los propietarios de hoteles ofrecerán en los próximos 5 años oportunidades de capacitación para el desarrollo de habilidades y destrezas del personal

$3 \quad$ Poco interés por parte de los colaboradores en ofrecer un servicio y atención al cliente de calidad

H3 = En los próximos 5 años se logrará que los colaboradores ofrezcan un servicio y atención de calidad

4

Falta de incentivos y de promoción para que el colaborador se sienta motivado

$\mathrm{H} 4=$ En los próximos 5 años el sector hotelero dispondrá de incentivos y de promoción para que sus colaboradores se sientan motivados

En la tabla $\mathrm{N}^{\mathrm{o}} 4$ se muestran los cuatro (4) problemas claves identificados en los análisis realizados y para cada uno de ellos se formularon las hipótesis correspondientes, tratando de describir correctamente su evolución futura y más adelante se plantean las recomendaciones correspondientes. 
En esta tabla $\mathrm{N}^{0} 5$ se busca identificar qué tan o no es probable cada una de las hipótesis de acuerdo a la opinión de los expertos entrevistados. La ponderación que se utilizará será:

$1=$ Es un hecho muy poco probable $(10 \%)$

$2=$ Es un hecho poco probable $(30 \%)$

$3=$ Es un hecho con probabilidad media (50\%)

$4=$ Es un hecho bastante probable $(70 \%)$

$5=$ Es un hecho muy probable $(90 \%)$

\section{Tabla $\mathbf{N}^{\circ} 5$}

\section{EL MÉTODO DE ESCENARIO}

\begin{tabular}{|l|l|}
\hline $\begin{array}{l}\text { 1= Es un hecho muy poco probable } \\
\text { 2= Es un hecho poco probable }\end{array}$ & $10 \%$ \\
$3=$ Es un hecho con probabilidad med & $30 \%$ \\
\hline
\end{tabular}

\begin{tabular}{|c|c|c|c|c|c|c|c|c|c|c|c|c|c|c|}
\hline Experto & H1 & $\mathrm{H} 1 / \mathrm{H} 2+$ & $\mathrm{H} 1 / \mathrm{H} 3+$ & $\mathrm{H} 1 / \mathrm{H} 4+$ & H1/H2 - & H1/H3 - & H1/H4 - & $\mathrm{H} 2$ & $\mathrm{H} 2 / \mathrm{H} 1+$ & $\mathrm{H} 2 / \mathrm{H} 3+$ & $\mathrm{H} 2 / \mathrm{H} 4+$ & H2/H1- & H2/H3- & $\mathrm{H} 2 / \mathrm{H} 4-$ \\
\hline 1 & 3 & 3 & 3 & 3 & 1 & 1 & 1 & 4 & 4 & 4 & 4 & 4 & 1 & 1 \\
\hline 2 & 4 & 4 & 4 & 4 & 2 & 2 & 2 & 5 & 4 & 3 & 3 & 4 & 3 & 3 \\
\hline 3 & 3 & 3 & 4 & 4 & 3 & 3 & 3 & 3 & 3 & 2 & 2 & 3 & 3 & 3 \\
\hline 4 & 4 & 4 & 4 & 3 & 3 & 3 & 2 & 4 & 3 & 4 & 4 & 3 & 3 & 3 \\
\hline 5 & 3 & 3 & 4 & 3 & 5 & 5 & 3 & 4 & 3 & 4 & 2 & 2 & 1 & 1 \\
\hline 6 & 4 & 3 & 4 & 3 & 3 & 3 & 2 & 3 & 3 & 3 & 3 & 2 & 2 & 2 \\
\hline 7 & 3 & 3 & 3 & 3 & 1 & 1 & 1 & 4 & 3 & 2 & 4 & 4 & 2 & 2 \\
\hline Moda & 3 & 3 & 4 & 3 & 3 & 3 & 2 & 4 & 3 & 4 & 4 & 4 & 3 & 3 \\
\hline Experto & $\mathrm{H3}$ & $\mathrm{H} 3 / \mathrm{H} 1+$ & $\mathrm{H} 3 / \mathrm{H} 2+$ & $\mathrm{H} 3 / \mathrm{H} 4+$ & H3/H1 - & H3/H2 - & $\mathrm{H} 3 / \mathrm{H} 4-$ & $\mathrm{H} 4$ & $\mathrm{H} 4 / \mathrm{H} 1+$ & $\mathrm{H} 4 / \mathrm{H} 2+$ & $\mathrm{H} 4 / \mathrm{H} 3+$ & H4/H1 - & $\mathrm{H} 4 / \mathrm{H}_{2}-$ & H4/H3 - \\
\hline 1 & 3 & 3 & 4 & 4 & 2 & 2 & 2 & 4 & 4 & 3 & 3 & 4 & 2 & 2 \\
\hline 2 & 4 & 4 & 3 & 3 & 2 & 2 & 2 & 5 & 4 & 3 & 3 & 4 & 3 & 3 \\
\hline 3 & 3 & 3 & 4 & 4 & 3 & 3 & 3 & 3 & 3 & 2 & 3 & 3 & 3 & 3 \\
\hline 4 & 4 & 3 & 3 & 3 & 3 & 3 & 2 & 4 & 3 & 4 & 4 & 3 & 3 & 3 \\
\hline 5 & 3 & 3 & 4 & 3 & 5 & 5 & 3 & 4 & 3 & 4 & 2 & 2 & 2 & 2 \\
\hline 6 & 4 & 3 & 4 & 3 & 3 & 3 & 2 & 3 & 3 & 3 & 3 & 2 & 2 & 2 \\
\hline 7 & 3 & 3 & 3 & 3 & 2 & 2 & 2 & 4 & 3 & 3 & 4 & 4 & 2 & 2 \\
\hline Moda & 3 & 3 & 4 & 3 & 3 & 3 & 2 & 4 & 3 & 3 & 3 & 4 & 2 & 2 \\
\hline
\end{tabular}




\section{Tabla $\mathbf{N}^{\circ} 6$}

\begin{tabular}{|c|c|c|c|c|c|}
\hline \multicolumn{6}{|c|}{ EL MÉTODO DE ESCENARIO } \\
\hline HIPÓTESIS & OPTIMISTA & MODERADO & PESIMISTA & $\begin{array}{c}\text { PROBABILIDAD } \\
\text { DE OCURRENCIA } \\
\text { ABSOLUTA }\end{array}$ & $\begin{array}{l}\text { PROBABILIDAD DE } \\
\text { OCURRENCIA } \\
\text { CONDICIONADA } \\
\end{array}$ \\
\hline $\mathrm{H} 2$ & \begin{tabular}{|l|} 
Todos los propietarios \\
de hoteles ofrecerán en \\
los próximos 5 años \\
oportunidades de \\
capacitación para el \\
desarrollo de habilidades \\
y destrezas del personal
\end{tabular} & & & $70 \%$ & $\begin{array}{c}\text { Si se cumple: } \\
\text { H1, H3 Y H4 }=70 \% \\
\text { Si no se cumple: H1, } \\
\text { H3 Y H4 } 4=70 \%\end{array}$ \\
\hline $\mathrm{H} 4$ & $\begin{array}{l}\text { En los próximos } 5 \text { el } \\
\text { Sector Hotelero } \\
\text { dispondrá de incentivos y } \\
\text { de promoción para que } \\
\text { sus colaboradores se } \\
\text { sientan motivados. }\end{array}$ & & & $70 \%$ & $\begin{array}{l}\text { Si se cumple: } \\
\text { H1, H2 Y H3 }=70 \% \\
\text { Si no se cumple: } \\
\text { H1, H2 Y H3 }=70 \%\end{array}$ \\
\hline H1 & & $\begin{array}{l}\text { En los próximos } 5 \text { años } \\
\text { el Sector Hotelero } \\
\text { contará con una alta } \\
\text { satisfacción laboral y } \\
\text { motivación por parte de } \\
\text { los colaboradores. }\end{array}$ & & $50 \%$ & $\begin{array}{c}\text { Si se cumple: } \\
\text { H2, H3 Y H4 =50\% } \\
\text { Si no se cumple: } \\
\text { H2, H3 Y H4 }=50 \%\end{array}$ \\
\hline H3 & & $\begin{array}{l}\text { En los próximos } 5 \text { años } \\
\text { se logrará que los } \\
\text { colaboradores ofrezcan } \\
\text { un servicio y atención } \\
\text { de calidad } \\
\end{array}$ & & $50 \%$ & $\begin{array}{l}\text { Si se cumple: } \\
\text { H1, H2 Y H4 =50\% } \\
\text { Si no se cumple: } \\
\text { H1, H2 Y H4 }=50 \%\end{array}$ \\
\hline
\end{tabular}

Las hipótesis 2 y 4 muestran un nivel de probabilidad de ocurrencia absoluta, por lo que se establecen en el área optimista con un 70\% de probabilidad. Las hipótesis 1 y 3 muestran un nivel de probabilidad de ocurrencia condicionada, por lo que se establecen en el área moderada con un $50 \%$ de probabilidad.

\section{Discusión}

Panamá no cuenta con una política clara en turismo pese a la amplia oferta y recursos que posee a mano. No nos hemos enfocado en definir qué es Panamá (como producto turístico), no tenemos una estrategia clara y no nos ponemos de acuerdo.

Los costos de venir a Panamá se han incrementado. Y paulatinamente, se ha dado una baja ocupación hotelera desde el año 2017 de aproximadamente 50\% y este es un diagnóstico de cómo se está moviendo la industria del turismo. En la recién campaña electoral los distintos candidatos a la presidencia del país observaron que una tarea a atender es el desarrollo del turismo.

Desde el año 2010 se vio venir este problema y se notó un cambio en los siguientes años. Hubo un crecimiento muy grande, se apostaron a muchísimas cosas, pero no se ha centrado la atención a un tema tan primordial como lo es el servicio al cliente.

No debemos perder de vista que los clientes demandan y exigen una experiencia más auténtica donde la atención a sus necesidades sea la clave. A fin de poder llegar a acciones concretas que ayuden a mejorar las deficiencias que se tienen en cuanto a la calidad del servicio al cliente, se han identificado que los cuatro (4) problemas claves que se ubicaron el área de conflicto: Baja satisfacción laboral y motivación por parte de los colaboradores. Propietarios no ofrecen oportunidades de capacitación 
para el desarrollo de habilidades y destrezas del personal. Poco interés por parte de los colaboradores en ofrecer un servicio y atención al cliente de calidad. Falta de incentivos y de promoción para que el colaborador se sienta motivado.

Los actores claves que mencionamos a continuación se deben integrar y trabajar en forma conjunta y con acciones claras para atender, prioritariamente, los cuatros (4) problemas claves identificados en este trabajo y los demás mencionados por las personas que se entrevistaron para estos fines y los cuales se mencionan en la matriz de actores. Por parte del gobierno se espera se concrete algunas de las promesas de hacer con la atención, que la industria del turismo rinde a la economía del país como a sus partes actoras, un tema de prioridad.

\section{Recomendaciones}

- Que se promueva una mayor integración entre todos los actores del sector turismo que se muestran en este trabajo. Esto permitirá llegar a acuerdos, recomendaciones y/o acciones que brinden resultados inmediatos a la mejora del servicio al cliente en ciudad de Panamá, en los próximos cinco (5) años venideros.

- Que los actores del sector turismo traten como socios a sus colaboradores quienes también tienen intereses dentro de la empresa con la finalidad de lograr los mejores resultados para todos.

- Que se fomente el trabajo en equipo y la libertad e independencia para que los colaboradores puedan tomar decisiones bajo sus propios riesgos. Muchas empresas han implementado la estrategia de reconocer públicamente al "Empleado del Mes" y que, adicionalmente, las empresas escuchen a los colaboradores y los hagan sentir parte de ellas.

- Que las empresas involucradas en el sector turismo fomenten la capacitación del personal. No basta con que la gente tenga la mejor actitud, también debe tener la mejor formación. Se debe elaborar un plan anual de capacitación para todo el personal del negocio. Esto permitirá capacitar constantemente al personal en temas de servicio al cliente: hay que recordar que no basta una sola charla sobre el tema.

- Que los actores del sector turismo inviertan en que sus colaboradores aprendan, refuercen y perfeccionen el idioma inglés. Inclusive, que inviertan para que sus colaboradores aprendan un tercer idioma y de esta manera, puedan mejorar la comunicación con sus clientes no sólo a lo interno de la empresa si no a lo externo, sobre todo si son internacionales.

- Que las empresas seleccionen a los colaboradores claves para que promuevan la calidad en el servicio, otorgando nuevas responsabilidades o promoviendo un encargado especial para solucionar las fallas al momento del encuentro. Esto se llama "empoderar". No tiene sentido contratar personas de talento o invertir en capacitaciones y después limitar sus habilidades de servicio. 


\section{Bibliografía}

Autoridad de Turismo de Panamá (ATP) (2017). Boletín Estadístico 2017. Departamento de Estadística. Disponible:

http://www.atp.gob.pa/sites/default/files/documentos/resumen_ejecutivo_2017.pdf

Autoridad de Turismo de Panamá (ATP) (2018a). Resumen Estadístico Enero a Junio 2018. Departamento de Estadística. Disponible:

http://www.atp.gob.pa/sites/default/files/documentos/resumen_estadistico_de_enero_a_junio_20 18.pdf

Autoridad de Turismo de Panamá (ATP) (2018b). Oferta de Alojamiento 2008-2017. Departamento de Estadística. Disponible:

http://www.atp.gob.pa/sites/default/files/documentos/oferta_de_alojamiento.pdf

Gabiña, J. (1998), La Prospectiva una Herramienta Cargada de Futuro. Editorial

Prentice Hall.

Garza, V. J. V. y Cortés, A. D. V. (2011). El uso del método MICMAC y MACTOR análisis prospectivo en un área operativa para la búsqueda de la excelencia operativa a través del Lean Manufacturing. Recuperado Octubre 2012 de:

http://www.web.facpya.uanl.mx/rev_in/Revistas/8.2/A6.pdf

Godet, M. (1991), Prospectiva y Planificación Estratégica. S.G Editores. Barcelona.

Godet, M. (1999), De la anticipación a la Acción. Manual de Prospectiva Estratégica. Alfaomega Grupo Editor. México, DF.

Godet, M. (2003). La caja de herramientas de la prospectiva estratégica. Centro Lindavista. México. Recuperado en septiembre 2011 de: http://es.scribd.com/doc/134321467/Caja-de- HerramientasPlaneación-Estratégica

Godet, M. (2007). Prospectiva Estratégica: problemas y métodos. Enero 2007. Cuaderno de LIPSOR. Recuperado en Agosto 2011 de: http:/ /www.prospektiker.es/prospectiva/cajaherramientas-2007.pdf

Gómez, E. (2009). Barreras contra el buen servicio. Como conservar más clientes (la amabilidad es la clave). Argentina. El Cid Editor.

Gómez, E. (2009). Barreras contra el buen servicio. Buenos Aires: El Cid.

Hernández Sampieri, R.; Fernández, C. y Baptista, M. (2014). Metodología de la Investigación. 6ta. Edición. México.

Kotler Philip, C. D. (2000). Dirección de Marketing. Edición de Milenio. Prentice Hall. 
Martínez-Tur, V.; Peiró, J. y Ramos, J. (2001). Calidad de servicio y satisfacción del cliente: Una perspectiva psicosocial. Síntesis. Madrid.

Medina, M. (2000). Futurica. Prospectiva en Acción. Colección Respuestas. IESAIC/UNESCO. Caracas.

Mi Diario. (2018). Revista Forbes destaca a Panamá como destino popular en 2018. Midiario.com, 29 de septiembre de 2018. Disponible en: https//www.midiariocom/panamá/nacionales/revistaforbes-destaca-panama-como-destino-popular-en-2018.

Nuñez, H. (2009). Servicio al cliente. México. Edamsa impresiones.

Rincón, S. y Mujica, N. (2004). Estudio prospectivo de la gestión tecnológica en las empresas del sector metalmecánico del estado Zulia. Revista Venezolana de Gerencia, Vol. 9, No. 26, abril-junio, 2004, pp. 289-314. Universidad del Zulia. Maracaibo, Venezuela.

Serna, H. (2006). Conceptos básicos. En Servicio al cliente. Colombia. Panamericana editorial Ltda.

Serna, P. (2006). Del servicio al cliente a la gestión de clientes. En Servicio al cliente. Colombia. Panamericana editorial Ltda.

Vidal, E. (2014). La calidad y su gestión en las organizaciones gallegas. Propuesta de un modelo de gestión de calidad total basado en el Modelo EFQM de Excelencia. Tesis Doctoral. Universidad de la Coruña. Disponible:

https://ruc.udc.es/dspace/bitstream/habdle/2183/12406/VidalV\%E1zqyez:_Estrella_TD_2014.p df?sequence $=4$

World Economic Forum (WEF) (2017). The Travel \& Tourism Competitiveness Report 2017. Geneva.

World Travel \& Tourism Council (WTTC) (2018). Travel \& Tourism Economic Impact 2018 World. Disponible: https://www.wttc.org/-/media/files/reports/economic-impactresearch/regions-2018/world2018.pdf 